\title{
THE TIME FRACTIONAL DIFFUSION EQUATION AND THE ADVECTION-DISPERSION EQUATION
}

\author{
F. HUANG ${ }^{12}$ and F. LIU 13
}

(Received 30 October, 2003; revised 21 June, 2004)

\begin{abstract}
The time fractional diffusion equation with appropriate initial and boundary conditions in an $n$-dimensional whole-space and half-space is considered. Its solution has been obtained in terms of Green functions by Schneider and Wyss. For the problem in whole-space, an explicit representation of the Green functions can also be obtained. However, an explicit representation of the Green functions for the problem in half-space is difficult to determine, except in the special cases $\alpha=1$ with arbitrary $n$, or $n=1$ with arbitrary $\alpha$. In this paper, we solve these problems. By investigating the explicit relationship between the Green functions of the problem with initial conditions in whole-space and that of the same problem with initial and boundary conditions in half-space, an explicit expression for the Green functions corresponding to the latter can be derived in terms of Fox functions. We also extend some results of Liu, Anh, Turner and Zhuang concerning the advection-dispersion equation and obtain its solution in half-space and in a bounded space domain.
\end{abstract}

\section{Introduction}

Applications of fractional derivatives can be dated back to the 19th century. For example, Caputo and Mainardi found good agreement with experimental results when using fractional derivatives for the description of viscoelastic materials [10, 11]. More recently, the idea has emerged that the space and/or time fractional partial differential equation, obtained from the standard partial differential equation that replaces the space-derivative and/or time-derivative by a fractional derivative, may more accurately describe some physical problems than the corresponding standard partial differential equation. More and more works by researchers from various fields of science and

\footnotetext{
${ }^{1}$ Department of Mathematics, Xımen University, Xiamen 361005, China; e-mail: fwliu@xmu.edu.cn. ${ }^{2}$ School of Mathematical Sciences, South China University of Technology, Guangzhou 510640, China; e-mail: huangfh@scut.edu.cn.

${ }^{3}$ School of Mathematical Sciences, Queensland University of Technology, QId 4001, Australia; e-mail: f.liu@qut.edu.au.

(C) Australian Mathematical Society 2005, Serial-fee code 1446-1811/05
} 
engineering deal with dynamical systems described by fractional-order differential equations (FDES), which have been used to represent many natural processes in physics $[8,28,33]$, finance $[25,27]$ and hydrology $[5,7]$. For example, the fractional diffusion equation (TFDE) and the fractional advection-dispersion equation (TFADE) have been widely researched $[6,12,17,30]$. From a physical viewpoint, they are obtained from a fractional Fick law replacing the classical Fick law, which describes transport processes with a long memory [16]. Nigmatullin [22, 23] pointed out that many of the universal electromagnetic, acoustic and mechanical responses can be modelled accurately using fractional diffusion-wave equations. For example, a TFDE has been explicitly introduced in physics by Nigmatullin [23] to describe diffusion in special types of porous media which exhibit a fractal geometry. Ginoa et al. [13] also presented a TFDE describing relaxation phenomena in complex viscoelastic materials. Roman and Alemany [26] investigated continuous-time random walks on fractals. Gorenflo et al. [16] generated discrete random models suitable for simulating random variables whose spatial probability density evolves in time according to a TFDE.

Wyss [32] and Schneider and Wyss [29] considered the time fractional diffusion and wave equations and obtained the solution in closed form in terms of Fox functions. Other research has considered the same equation and space and/or time FDES for different motives. Recently, Anh and Leonenko also considered the same fractional diffusion-wave equations [3, 4] and the heat equation [2]. Gorenflo, Luchko and Mainardi [14] used the similarity method and the Laplace transform method to obtain the scale-invariant solution of the time fractional diffusion-wave equation in terms of the Wright function. Agrawal presented a general solution for a fractional diffusionwave equation defined in a bounded space domain by the finite sine and Laplace transform technique [1]. Gorenflo and Mainardi considered random walk models for space fractional diffusion processes [15]. The space-time fractional diffusion equation has also been treated by Mainardi, Luchko and Pagnini as a Cauchy problem and its fundamental solution (or Green function) was investigated with respect to its scaling and similarity properties [21]. Benson, Whearcraft and Meerschaert [6] considered the space fractional advection-dispersion equation and gave an analytical solution featuring the $\alpha$-stable error function. Liu et al. [18] considered the space fractional Fokker-Planck equation and presented its numerical solution. Recently, Liu et al. [19] also treated the TFADE and derived the complete solution of this equation with an initial condition. Many other FDEs, such as the Black-Scholes equation, also were considered. Professor Meerschaert (http://unr.edu/homepage/mcubed/) has pointed out that fractional derivatives are almost as old as their integer-order cousins. Recently, fractional derivatives have found new applications in engineering, physics, finance and hydrology. In physics, fractional derivatives are used to model anomalous diffusion, where a could of particles spreads differently than the classical Brownian 
motion model predicts.

The results presented in this paper are based on the works of Schneider and Wyss [29] and Liu et al. [19].

\section{The time fractional diffusion equation}

We consider the equation

$$
\frac{\partial^{\alpha} u(\mathrm{x}, t)}{\partial t^{\alpha}}=\Delta u(\mathrm{x}, t), \quad 0<\alpha \leq 1,
$$

with the fractional derivative defined in the Caputo sense [9]:

$$
\frac{d^{\alpha} \varphi(t)}{d t^{\alpha}}= \begin{cases}\frac{d^{n} \varphi(t)}{d t^{n}}, & \alpha=n \in \mathbb{N}, \\ \frac{1}{\Gamma(n-\alpha)} \int_{0}^{t}(t-\tau)^{n-\alpha-1} \frac{d^{n} \varphi(\tau)}{d \tau^{n}} d \tau, & n-1<\alpha<n,\end{cases}
$$

where $\varphi$ is a continuous function. Properties and more details about Caputo's fractional derivative can be found in $[20,24]$. The Laplace transform of a function $\varphi(t)$, $0<t<\infty$, is defined as follows $[20,24]$ :

$$
\tilde{\varphi}(p)=\mathscr{L}\{\varphi(t)\}=\mathscr{L}\{\varphi(t) ; p\}=\int_{0}^{\infty} e^{-p t} \varphi(t) d t
$$

There is a fundamental formula

$$
\mathscr{L}\left\{\frac{d^{\alpha} \varphi(t)}{d t^{\alpha}}, p\right\}=p^{\alpha} \tilde{\varphi}(p)-\sum_{k=0}^{n-1} p^{\alpha-1-k} \varphi^{(k)}\left(0^{+}\right), \quad n-1<\alpha \leq n, n \in \mathbb{N} .
$$

We refer to (2.1) as the time fractional diffusion equation (TFDE). For this equation, there are a series of papers (see [20] and the references therein) which consider the following two basic problems, which are initial value problems (IVPs) or initial boundary-value problems (IBVP) respectively:

Problem I: The TFDE in a whole-space (IVP)

$$
\begin{aligned}
u\left(\mathbf{x}, 0^{+}\right) & =f(\mathbf{x}), & & \mathbf{x} \in \mathbb{R}^{n}, \\
u\left(\mathbf{x}^{T}, \mp \infty, t\right) & =0, & & t>0 ;
\end{aligned}
$$

Problem II: The TFDE in a half-space (IBVP)

$$
\begin{aligned}
u\left(\mathrm{x}, 0^{+}\right) & =f(\mathbf{x}), & & \mathrm{x} \in \mathbb{D}, \\
\lambda u\left(\mathbf{x}^{0}, t\right)-\mu \frac{\partial u}{\partial x_{n}}\left(\mathbf{x}^{0}, t\right) & =v\left(\mathbf{x}^{T}, t\right), & & t>0 \\
u\left(\mathbf{x}^{T},+\infty, t\right) & =0, & & t>0
\end{aligned}
$$


with given sufficiently well-behaved functions $f$ and $v$. The notation is as follows:

$$
\begin{aligned}
& \mathbb{D}=\mathbb{R}^{n-1} \times \mathbb{R}_{+}, \quad \partial \mathbb{D}=\mathbb{R}^{n-1} \times\{0\}, \quad \mathbf{x}=\left(x_{1}, x_{2}, \ldots, x_{n}\right) \in \mathbb{D}, \\
& \mathbf{x}^{T}=\left(x_{1}, x_{2}, \ldots, x_{n-1}\right) \in \mathbb{R}^{n-1}, \quad \mathbf{x}^{0}=\left(x_{1}, x_{2}, \ldots, x_{n-1}, 0\right) \in \partial \mathbb{D} .
\end{aligned}
$$

For problem I, the solution has been obtained by Schneider and Wyss [29]:

$$
u(\mathbf{x}, t)=\int_{\mathbb{R}^{n}} d^{\mathbf{n}} \mathbf{y} G^{\alpha}(|\mathbf{x}-\mathbf{y}|, t) f(\mathbf{y}),
$$

where $G^{\alpha}(x, t)$ is referred to as the Green function or fundamental solution, which is intended to be the solution for the IVP corresponding to the initial condition $f(\mathbf{x})=\delta(\mathbf{x})$ (where $\delta$ denotes the Dirac delta distribution):

$$
G^{\alpha}(r, t)=\alpha^{-1} \pi^{-n / 2} r^{-n} H_{12}^{20}\left(2^{-2 / \alpha} r^{2 / \alpha} t^{-1} \mid \begin{array}{c}
(1,1) \\
(n / 2,1 / \alpha),(1,1 / \alpha)
\end{array}\right),
$$

where $H_{p q}^{m n}(z)$ is a Fox function (or $H$-function) [24, 31].

For problem II, as the propagation equation (2.1) is linear, it is sufficient to consider separately the problems

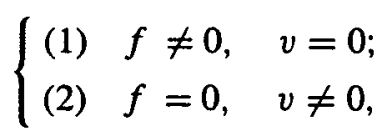

which Schneider and Wyss called first and second type problems respectively. They also obtained solutions in terms of their corresponding Green functions. However, they only derived an explicit representation of the Green functions for the first type problem and that for the second type problem for some special cases. In fact, the second type problem can be further subdivided by the choice of $\lambda, \mu$ in (2.7):

$$
\begin{cases}\text { (1) } \lambda=1, & \mu=0 ; \\ \text { (2) } \lambda=0, & \mu=1 ; \\ \text { (3) } \lambda \neq 0, & \mu=1 .\end{cases}
$$

Then their solutions may be expressed [29] as

$$
u(\mathbf{x}, t)=\int_{\mathbb{R}^{n-1}} d^{n-1} \mathbf{y} \int_{0}^{t} d \tau G_{j}^{\alpha}\left(\left(\mathbf{x}^{T}-\mathbf{y}, x_{n}\right), t-\tau\right) v(\mathbf{y}, \tau),
$$

where the indices $j=1,2,3$ correspond to the cases $(j)$ of (2.10). However, Schneider and Wyss [29] did not succeed in finding explicit representations for $G_{j}^{\alpha}$ except in the special cases $\alpha=1$ with arbitrary $n$, or $n=1$ with arbitrary $\alpha$. For the special case when $\alpha$ is set to be one, they found that $G_{j}^{\alpha}(j=1,2)$ are non-negative. They 
then wanted to know whether this remains true for arbitrary $n$ with $0<\alpha<1$. They pointed out that this question remained open for $n>1$.

Based on the work of Schneider and Wyss, we try to resolve the above questions. In other words, we provide explicit representations for $G_{j}^{\alpha}(j=1,2)$ and show that they are indeed non-negative for arbitrary $n$ and $0<\alpha \leq 1$.

2.1. The TFDE in one spatial dimension In order to motivate the technique and for simplicity, we first focus our attention on (2.1) in one dimension, that is,

$$
\frac{\partial^{\alpha} u(x, t)}{\partial t^{\alpha}}=\frac{\partial^{2} u(x, t)}{\partial x^{2}}, \quad 0<\alpha \leq 1, t>0 .
$$

The following basic initial and boundary conditions are considered:

(a) The Cauchy problem (IVP)

$$
\begin{aligned}
u\left(x, 0^{+}\right) & =f(x), & -\infty<x<+\infty \\
u(\mp \infty, t) & =0, & t>0
\end{aligned}
$$

(b) The signalling problem (IBVP)

$$
\begin{aligned}
u\left(x, 0^{+}\right) & =0, & & x>0 \\
u\left(0^{+}, t\right)=g(t), & u(+\infty, t) & =0, & t>0 .
\end{aligned}
$$

The solutions of the Cauchy and signalling problems were derived respectively [29] to be

and

$$
u(x, t)=\int_{-\infty}^{+\infty} d y G_{c}^{\alpha}(|x-y|, t) f(y)
$$

$$
u(x, t)=\int_{0}^{t} d \tau G_{s}^{\alpha}(x, t-\tau) g(\tau),
$$

where $G_{c}^{\alpha}$ and $G_{s}^{\alpha}$ are the corresponding Green functions which represent the socalled fundamental solutions, obtained when $f(x)$ is set to be $\delta(x)$ or $g(t)$ to be $\delta(t)$ respectively. It should be noted that $G_{c}^{\alpha}(x, t)=G_{c}^{\alpha}(|x|, t)$ since the Green function is an even function of $x$.

Applying the Laplace transform (2.2) to (2.12) with respect to variable $t$ and using the formula (2.3) with (2.13) for $f(x)=\delta(x)$, we obtain

$$
p^{\alpha} \widetilde{G}_{r}^{\alpha}(x, p)-\delta(x) p^{\alpha-1}=\frac{\partial^{2}}{\partial x^{2}} \widetilde{G}_{r}^{\alpha}(x, p)
$$


Because of the singular term $\delta(x)$, we have to consider the above equation separately in the two intervals $x<0$ and $x>0$, imposing the natural boundary conditions (2.14) and the necessary matching conditions at $x=0$. Then we obtain

$$
\widetilde{G}_{c}^{\alpha}(x, p)=\frac{p^{\alpha / 2-1}}{2} e^{-p^{\alpha / 2}|x|}
$$

For the signalling problem, the same application of the Laplace transform to (2.12) with (2.15) leads to

$$
\widetilde{G}_{s}^{\alpha}(x, p)=e^{-x p^{\alpha / 2}}=e^{-\left(x^{2 / \alpha} p\right)^{\alpha / 2}}=\widetilde{w}_{\alpha / 2}\left(x^{2 / \alpha} p\right) .
$$

Returning to the time domain and letting $z=x^{-2 / \alpha} t$, we obtain the solution

$$
G_{s}^{\alpha}(x, t)=x^{-2 / \alpha} w_{\alpha / 2}\left(x^{-2 / \alpha} t\right)=z t^{-1} w_{\alpha / 2}(z)
$$

Here $w_{\beta}(0<\beta<1)$ denotes the one-sided stable (or Lévy) probability density [29] characterised by its Laplace transform $\widetilde{w}_{\beta}(p)=e^{-p^{\beta}}$. In fact, it can be explicitly expressed [29] as

$$
w_{\beta}(x)=\beta^{-1} x^{-2} H_{11}^{10}\left(x^{-1} \mid \begin{array}{c}
(-1,1) \\
(-1 / \beta, 1 / \beta)
\end{array}\right) .
$$

From (2.17) and (2.18), we recognise that for $x>0$,

$$
\frac{\partial}{\partial p} \widetilde{G}_{s}^{\alpha}(x, p)=-\alpha x \tilde{G}_{c}^{\alpha}(x, p),
$$

and in the time domain we obtain the relation

$$
x G_{c}^{\alpha}(x, t)=\frac{t}{\alpha} G_{s}^{\alpha}(x, t), \quad x_{n}>0 .
$$

Then we can obtain the explicit representation for $G_{c}^{\alpha}$ with $0<\alpha \leq 1$

$$
G_{c}^{\alpha}(x, t)=\frac{z}{\alpha x} w_{\alpha / 2}(z)=\alpha^{-1} t x^{-1-\alpha / 2} w_{\alpha / 2}\left(t x^{-2 / \alpha}\right),
$$

which is in agreement with the known result in [29].

REMARK 1 . We can easily show that $G_{c}^{\alpha}$ is a probability on $\mathbb{R}$, for $G_{c}^{\alpha}(x, t)=$ $G_{c}^{\alpha}(|x|, t)$ and that $w_{\beta}(z)$ is a probability on $\mathbb{R}_{+}$.

REMARK 2. We can obtain similar results for $1<\alpha \leq 2$ if we add another initialvalue condition $(\partial u / \partial t)(x, 0)=0$. 
2.2. The TFDE in an $n$-dimensional half-space Using a similar method to that outlined above, we can treat (2.1) in an $n$-dimensional space, that is to say, we will analyse the Green function in (2.11) corresponding to the second type of problem II. We use $\bar{g}\left(k, x_{n}, p\right)$ to denote a Fourier transform with respect to variable $\mathbf{x}^{T}$ and a Laplace transform with respect to variable $t$ for the function $g(x, t)$, that is,

$$
\bar{g}\left(k, x_{n}, p\right)=\int_{\mathbb{R}^{n-1}} d^{n-1} \mathbf{x}^{T} e^{i k \mathbf{x}^{T}} \int_{0}^{+\infty} d t e^{-p t} g(t) .
$$

For problem I, the Fourier-Laplace transform for (2.1) with the initial condition (2.4) for $f(\mathbf{x})=\delta(\mathbf{x})$ leads to

$$
p^{\alpha} \bar{G}^{\alpha}-p^{\alpha-1} \delta\left(x_{n}\right)=-k^{2} \bar{G}^{\alpha}+\frac{\partial^{2}}{\partial x_{n}^{2}} \bar{G}^{\alpha} .
$$

Taking into account the boundary condition (2.5) and the necessary matching conditions at $x_{n}=0$, we derive

$$
\bar{G}^{\alpha}\left(k, x_{n}, p\right)=\frac{p^{\alpha-1}}{2 \sqrt{p^{\alpha}+k^{2}}} e^{-\sqrt{p^{\alpha}+k^{2}}\left|x_{n}\right|} .
$$

For problem II, the application of the Fourier-Laplace transform to (2.1) with (2.6), where $f(\mathbf{x})=0$, leads to $\partial^{2} \bar{G}_{j}^{\alpha} / \partial x_{n}^{2}=\left(p^{\alpha}+k^{2}\right) \bar{G}_{j}^{\alpha}$, with the general solution

$$
\bar{G}_{J}^{\alpha}\left(k, x_{n}, p\right)=A(k, p) e^{-\sqrt{p^{a}+k^{2} x_{n}}}+B(k, p) e^{+\sqrt{p^{a}+k^{2} x_{n}}} .
$$

The coefficients $A$ and $B$ are determined by the conditions (2.7)-(2.8) with $v\left(\mathbf{x}^{T}, t\right)=$ $\delta\left(\mathbf{x}^{T}\right) \delta(t)$, that is, $\bar{G}_{1}^{\alpha}(k, 0, p)=1$ and $\partial \bar{G}_{2}^{\alpha}(k, 0, p) / \partial x_{n}=-1$. Consequently,

$$
\begin{aligned}
& \bar{G}_{1}^{\alpha}\left(k, x_{n}, p\right)=e^{-\sqrt{p^{\alpha}+k^{2} x_{n}}} \text { and } \\
& \bar{G}_{2}^{\alpha}\left(k, x_{n}, p\right)=\frac{1}{\sqrt{p^{\alpha}+k^{2}}} e^{-\sqrt{p^{\alpha}+k^{2} x_{n}}} .
\end{aligned}
$$

From (2.19)-(2.21), we recognise that for $x_{n}>0$,

$$
\begin{aligned}
& \frac{\partial}{\partial p} \bar{G}_{1}^{\alpha}\left(k, x_{n}, p\right)=-\alpha x_{n} \bar{G}^{\alpha}\left(k, x_{n}, p\right) \quad \text { and } \\
& \frac{\partial}{\partial x_{n}} \bar{G}_{2}^{\alpha}\left(k, x_{n}, p\right)=-\bar{G}_{1}^{\alpha}\left(k, x_{n}, p\right) .
\end{aligned}
$$

Returning to the space-time domain we obtain the relation

$$
\begin{aligned}
x_{n} G^{\alpha}(\mathbf{x}, t) & =\frac{t}{\alpha} G_{1}^{\alpha}(\mathbf{x}, t), & x_{n}>0 \\
G_{2}^{\alpha}(\mathbf{x}, t) & =\int_{x_{n}}^{\infty} d y G_{1}^{\alpha}\left(\mathbf{x}^{T}, y, t\right), & x_{n}>0
\end{aligned}
$$


First, we can obtain an explicit representation for $G_{1}^{\alpha}$,

$$
\begin{aligned}
G_{1}^{\alpha}(\mathbf{x}, t) & =\alpha x_{n} t^{-1} G^{\alpha}(\mathbf{x}, t) \\
& =2^{-1} \alpha x_{n} t^{-1} \pi^{-n / 2} \mathbf{x}^{-n} H_{12}^{20}\left(2^{-1} \mathbf{x} t^{-\alpha / 2} \mid \begin{array}{c}
(1, \alpha / 2) \\
(n / 2,1 / 2),(1,1 / 2)
\end{array}\right) .
\end{aligned}
$$

Then an explicit representation for $G_{2}^{\alpha}$ follows from (2.23).

It is obvious that for $j=1,2$,

$$
G_{j}^{\alpha}(\mathbf{x}, t) \geq 0, \quad \mathbf{x} \in \mathbb{D}, t>0 .
$$

For the particular choices that $\alpha=1$ or $n=1$, the results are in good agreement with those in [29].

\section{The time fractional advection-dispersion equation}

As an application of the derived theory we extend the above analysis to the time fractional advection-dispersion equation (TFADE) which Liu et al. [19] investigated recently. Furthermore, we seek the solution for the TFADE in a bounded domain using the same approach as in [1]. The TFADE is obtained by replacing the time derivative in the advection-dispersion equation by a generalised derivative of order $\alpha$ with $0<\alpha \leq 1$ :

$$
\frac{\partial^{\alpha} C(x, t)}{\partial t^{\alpha}}=-\nu \frac{\partial C(x, t)}{\partial x}+D \frac{\partial^{2} C(x, t)}{\partial x^{2}}, \quad t>0
$$

with the initial condition

$$
C(x, 0)=C_{0}(x),
$$

where $\nu \geq 0, D>0$ and $\partial^{\alpha} C(x, t) / \partial t^{\alpha}$ is a Capato's fractional derivative. This system can be expressed by the following integral equation:

$$
C(x, t)=C(x, 0)+\frac{1}{\Gamma(\alpha)} \int_{0}^{t}(t-\tau)^{\alpha-1}\left[-\nu \frac{\partial C(x, \tau)}{\partial x}+D \frac{\partial^{2} C(x, \tau)}{\partial x^{2}}\right] d \tau
$$

with $n-1<\alpha \leq n, n=1$. To reduce the above equation to a more familiar form, let

$$
C(x, t)=u(\xi, t) \exp \left(\frac{\nu \xi}{2 \sqrt{D}}\right), \quad \xi=\frac{x}{\sqrt{D}},
$$

with the initial condition

$$
C(x, 0)=C_{0}(x)=u(\xi, 0) \exp \left(-\frac{\nu \xi}{2 \sqrt{D}}\right) .
$$


The TFADE is then reduced [19] to

$$
\begin{aligned}
u(\xi, t) & =u(\xi, 0)+\frac{1}{\Gamma(\alpha)} \int_{0}^{t}(t-\tau)^{\alpha-1}\left[\frac{\partial^{2} u(\xi, \tau)}{\partial \xi^{2}}-\mu^{2} u(\xi, \tau)\right] d \tau \\
& =u(\xi, 0)+D^{-\alpha}\left[\frac{\partial^{2} u(\xi, t)}{\partial \xi^{2}}-\mu^{2} u(\xi, t)\right]
\end{aligned}
$$

and

$$
u(\xi, 0)=C_{0}\left(\frac{\xi}{\sqrt{D}}\right) \exp \left(-\frac{\nu \xi}{2 \sqrt{D}}\right)
$$

where $\mu^{2}=v^{2} / 4 D, D^{-\alpha}$ is the fractional integral of order $\alpha$ [24] and its Laplace transform is given by

$$
\mathscr{L}\left\{D^{-\alpha} \varphi(t)\right\}=\frac{1}{\Gamma(\alpha)} \mathscr{L}\left\{t^{\alpha-1}\right\} \mathscr{L}\{\varphi(t)\}=p^{-\alpha} \tilde{\varphi}(p), \quad \alpha>0 .
$$

According to the properties of the Laplace and Mellin transforms and the Fox function, Liu et al. found the Green function for the reduced TFADE (3.4)-(3.5) in the half-space domain $(\xi \geq 0)$, that is,

where

$$
u(\xi, t)=\int_{0}^{\infty} G_{\mu}^{\alpha}(|\xi-y|, t) u(y, 0) d y
$$

$$
G_{\mu}^{\alpha}(r, t)=\frac{1}{2 \sqrt{\pi t^{\alpha}}} \int_{0}^{\infty} e^{-\mu^{2} t^{\alpha} \sigma-r^{2} /\left(4 t^{\alpha} \sigma\right)} H_{11}^{10}\left(\sigma \mid \begin{array}{c}
(1-3 \alpha / 2, \alpha) \\
(-1 / 2,1)
\end{array}\right) d \sigma .
$$

3.1. The solution of the TFADE in the half-space domain In fact, the above results (3.6) - (3.7) can easily be extended to the Cauchy problem for (3.1) in the whole-space domain with

$$
\begin{aligned}
C\left(x, 0^{+}\right) & =C_{0}(x), & -\infty<x<+\infty, \\
C(\mp \infty, t) & =0, & t>0,
\end{aligned}
$$

which corresponds to the reduced TFADE (3.4) for $\xi \in \mathbb{R}$ by the relation (3.3). The solution is obtained by using the same technique as that of Liu et al. [19]

$$
u(\xi, t)=\int_{-\infty}^{+\infty} G_{\mu r}^{\alpha}(|\xi-y|, t) u_{0}(y) d y .
$$

An explicit representation for $G_{\mu r}^{\alpha}$ is also obtained:

$$
G_{\mu c}^{\alpha}(r, t)=\frac{1}{2 \sqrt{\pi t^{\alpha}}} \int_{-\infty}^{+\infty} e^{-\mu^{2} t^{\alpha} \sigma-r^{2} /\left(4 t^{\alpha} \sigma\right)} H_{11}^{10}\left(\sigma \mid \begin{array}{c}
(1-3 \alpha / 2, \alpha) \\
(-1 / 2,1)
\end{array}\right) d \sigma
$$


with a Laplace transform of the form

$$
\widetilde{G}_{\mu c}^{\alpha}(\xi, p)=\frac{p^{\alpha-1}}{2 \sqrt{p^{\alpha}+\mu^{2}}} e^{-\sqrt{p^{\alpha}+\mu^{2}}|\xi|} .
$$

The signalling problem for (3.1) in the half-space domain is considered with

$$
\begin{aligned}
C\left(x, 0^{+}\right) & =0, & x>0, \\
C\left(0^{+}, t\right)=g(t), \quad C(+\infty, t) & =0, & t>0 .
\end{aligned}
$$

It corresponds to the reduced TFADE (3.4) for $\xi>0$ with

$$
\begin{aligned}
u\left(\xi, 0^{+}\right) & =0, & \xi>0, \\
u\left(0^{+}, t\right)=g(t), & u(+\infty, t)=0, & t>0 .
\end{aligned}
$$

The application of the Laplace transform to (3.4) with (3.9) gives

$$
\frac{\partial^{2} \widetilde{u}(\xi, p)}{\partial \xi^{2}}=\left(\mu^{2}+p^{\alpha}\right) \tilde{u}(\xi, p)
$$

with the general solution

$$
\tilde{u}(\xi, p)=A e^{-\sqrt{p^{\alpha}+\mu^{2}} \xi}+B e^{\sqrt{p^{\alpha}+\mu^{2} \xi}}
$$

Taking into account the boundary conditions (3.10), that is,

$$
\tilde{u}(0, p)=\widetilde{g}(p), \quad \tilde{u}(+\infty, p)=0,
$$

the coefficients $A$ and $B$ are determined, then

$$
\tilde{u}(\xi, p)=\widetilde{g}(p) e^{-\sqrt{p^{\alpha}+\mu^{2} \xi}}=\widetilde{g}(p) \widetilde{G}_{\mu s}^{\alpha}(\xi, p)=\mathscr{L}\left\{G_{\mu s}^{\alpha}(\xi, t) * g(t)\right\},
$$

where

$$
\widetilde{G}_{\mu s}^{\alpha}(\xi, p)=e^{-\sqrt{p^{\alpha}+\mu^{2} \xi}}
$$

The inverse Laplace transform of (3.11) gives

$$
u(\xi, t)=G_{\mu s}^{\alpha}(\xi, t) * g(t):=\int_{0}^{t} d \tau G_{\mu s}^{\alpha}(\xi, t-\tau) g(\tau) .
$$

From (3.12) and (3.8), we derive that for $r>0$,

$$
\frac{\partial}{\partial p} \widetilde{G}_{\mu s}^{\alpha}(r, p)=-\alpha r \widetilde{G}_{\mu r}^{\alpha}(r, p)
$$


Returning to the space-time domain we obtain the relation

$$
r G_{\mu c}^{\alpha}(r, t)=\frac{t}{\alpha} G_{\mu s}^{\alpha}(r, t), \quad r>0
$$

Consequently, we obtain explicit representations for $G_{\mu s}^{\alpha}$ as

$$
\begin{aligned}
G_{\mu s}^{\alpha}(r, t) & =\alpha r t^{-1} G_{\mu c}^{\alpha}(r, t) \\
& =\frac{\alpha r}{2 \sqrt{\pi t^{\alpha+2}}} \int_{-\infty}^{+\infty} e^{-\mu^{2} r^{\alpha} \sigma-r^{2} /\left(4 r^{\sigma} \sigma\right)} H_{11}^{10}\left(\sigma \mid \begin{array}{c}
(1-3 \alpha / 2, \alpha) \\
(-1 / 2,1)
\end{array}\right) d \sigma .
\end{aligned}
$$

3.2. The solution of the TFADE in a bounded domain In this section we seek the solution of the TFADE (3.1) in a bounded domain $0 \leq x \leq \sqrt{D} L$ with the initial condition (3.2). We further consider the following boundary conditions:

$$
C(0, t)=C(\sqrt{D} L, t)=0, \quad t \geq 0
$$

By the relation (3.3), we obtain another form of the reduced TFADE

$$
\frac{\partial^{\alpha} u(\xi, t)}{\partial t^{\alpha}}=-\mu^{2} u(\xi, t)+\frac{\partial^{2} u(\xi, t)}{\partial \xi^{2}}, \quad 0 \leq \xi \leq L, t>0
$$

with the boundary conditions

$$
u(0, t)=u(L, t)=0, \quad t \geq 0
$$

and initial condition (3.5). Taking the finite sine transform of (3.13), and applying the boundary conditions (3.14), we obtain

$$
\frac{\partial^{\alpha} \bar{u}(n, t)}{\partial t^{\alpha}}=-\mu^{2} \bar{u}(n, t)-(a n)^{\dot{2}} \bar{u}(n, t), \quad t>0
$$

where $a=\pi / L, n$ is a wave number and $\bar{u}(n, t)=\int_{0}^{L} u(y, t) \sin (a n y) d y$ is the finite sine transform of $u(y, t)$. The finite sine transform of $(3.5)$ is

$$
\bar{u}(n, 0)=\int_{0}^{\iota} C_{0}\left(\frac{\xi}{\sqrt{D}}\right) \exp \left(-\frac{\nu \xi}{2 \sqrt{D}}\right) \sin (\operatorname{an} \xi) d \xi
$$

Applying the Laplace transform to (3.15) and using the initial conditions (3.16), we obtain

$$
\overline{\bar{u}}(n, s)=\frac{s^{\alpha-1} \bar{u}(n, 0)}{s^{\alpha}+\mu^{2}+(a n)^{2}} .
$$


Noting the Laplace transform pair of the Mittag-Leffler function

$$
E_{\alpha}\left(c t^{\alpha}\right) \stackrel{\mathscr{L}}{\longleftrightarrow} \frac{p^{\alpha-1}}{p^{\alpha}-c}, \quad \Re(p)>|c|^{1 / \beta}, \quad c \in \mathscr{C},
$$

where the Mittag-Leffler function $E_{\alpha}(z)$ with $\alpha>0$ is defined by the following series representation, valid in the whole complex plane [24]:

$$
E_{\alpha}(z):=\sum_{n=0}^{\infty} \frac{z^{n}}{\Gamma(\alpha n+1)}, \quad z \in \mathscr{C} .
$$

We then obtain the pair

$$
\left.\frac{s^{\alpha-1} \bar{u}(n, 0)}{s^{\alpha}+\mu^{2}+(a n)^{2}} \stackrel{\mathscr{L}}{\longleftrightarrow} E_{\alpha}\left[-\left(\mu^{2}+(a n)^{2}\right) t^{\alpha}\right)\right] \bar{u}(n, 0) .
$$

Thus we can first apply the inverse Laplace transform and then the inverse finite sine transform for (3.17) to obtain

$$
\left.u(\xi, t)=\frac{2}{L} \sum_{n=1}^{\infty} E_{\alpha}\left[-\left(\mu^{2}+(a n)^{2}\right) t^{\alpha}\right)\right] \sin (a n \xi) \int_{0}^{L} C_{0}\left(\frac{\xi}{\sqrt{D}}\right) e^{-\nu \xi / 2 \sqrt{D}} \sin (\text { any }) d y .
$$

By the relation (3.3), we can obtain the solution $C(x, t)$.

\section{Conclusions}

We consider the time fractional diffusion equation and the advection-dispersion equation respectively in an $n$-dimensional whole- and half-space. We investigate the explicit relationships between the problems in whole-space with the corresponding problems in half-space using the Fourier-Laplace transform (2.19). Then fundamental solutions of the problems in half-space are obtained under the precondition that the fundamental solutions of the problems in the whole-space are known. We resolve some of the remaining problems stated in [29]. Furthermore, the time fractional advection-dispersion equation in a bounded space domain is also considered. Its solution is derived by the finite sine and Laplace transforms. In future research, we will consider their applications in hydrology and numerical simulation.

\section{Acknowledgements}

This research has been supported by the National Natural Science Foundation of China 10271098. 


\section{References}

[1] O. P. Agrawal, "Solution for a fractional diffusion-wave equation defined in a bounded domain", Nonlinear Dynam. 29 (2002) 145-155.

[2] V. V. Anh and N. N. Leonenko, "Non-Gaussian scenarios for the heat equation with singular initial conditions", Stochastic Processes Appl. 84 (1999) 91-1 14.

[3] V. V. Anh and N. N. Leonenko, "Scaling laws for fractional diffusion-wave equations with singular data", Statist. Probab. Let. Vol. 48 (2000) 239-252.

[4] V. V. Anh and N. N. Leonenko, "Harmonic analysis of random fractional diffusion-wave equations", Appl. Math. Comput. 141 (2003) 77-85.

[5] B. Baeumer, M. M. Meerschaert, D. A. Benson and S. W. Wheatcraft, "Subordinated advectiondispersion equation for contaminant transport", Water Resources Res. 37 (2001) 1543-1550.

[6] D. A. Benson, S. W. Wheatcraft and M. M. Meerschaert, "Application of a fractional advectiondispersion equation", Water Resources Res. 36 (2000) 1403-1412.

[7] D. A. Benson, S. W. Wheatcraft and M. M. Meerschaert, "The fractional-order governing equation of Lévy motion", Water Resources Res. 36 (2000) 1413-1424.

[8] J. P. Bouchaud and A. Georges, "Anomalous diffusion in disordered media-statistical mechanisms", Phys. Rep. 195 (1990) 127-293.

[9] M. Caputo, "Linear model of dissipation whose Q is almost frequency independent II", Geophys. J. Roy. Astr. Soc. 13 (1967) 529-539.

[10] M. Caputo, "Vibrations on an infinite viscoelastic layer with a dissipative memory", J. Acoust. Soc. Amer. 56 (1974) 897-904.

[11] M. Caputo and F. Mainardi, "A new dissipation model based on memory mechanism", Pure Appl. Geophysics 91 (1971) 134-147.

[12] A. Chaves, "Fractional diffusion equation to describe Lévy flights", Phys. Lett. A 239 (1998) 13-16.

[13] M. Ginoa, S. Cerbelli and H. E. Roman, "Fractional diffusion equation and relaxation in complex viscoelastic materials", Phys. A 191 (1992) 449-453.

[14] R. Gorenflo, Yu. Luchko and F. Mainardi, "Wright function as scale-invariant solutions of the diffusion-wave equation", J. Comp. Appl. Math. 118 (2000) 175-191.

[15] R. Gorenfio and F. Mainardi, "Random walk models for space-fractional diffusion processes", Fract. Calc. Appl. Anal. 1 (1998) 167-191.

[16] R. Gorenflo, F. Mainardi, D. Moretti and P. Paradisi, "Time fractional diffusion: a discrete random walk approach", Nonlinear Dynam. 29 (2002) 129-143.

[17] J. Klafter, A. Blumen and M. F. Shlesinger, "Stochastic pathways to anomalous diffusion", Phys. Rev. A 35 (1987) 3081-3085.

[18] F. Liu, V. V. Anh and I. Turner, "Numerical solution of the space fractıonal Fokker-Plank equation", J. Comput. Appl. Math. 166 (2004) 209-219.

[19] F. Liu, V. V. Anh, I. Turner and P. Zhuang, "Time fractional advection-dispersion equation", J. Appl. Math. Comput. 13 (2003) 223-245.

[20] F. Mainardi, "Fraction calculus: some basic problems in continuum and statistical mechanics", in Fractal and Fractional Calin Continuum Mechanics (eds. A. Carpinteri and F. Mainardi), (Springer, Wien, 1997) 291-348.

[21] F. Mainardi, Yu. Luchko and G. Pagnini, "The fundamental solution of the space-time fractional diffusion equation", Fract. Calc. Appl. Anal. 4 (2001) 153-192.

[22] R. R. Nigmatullin, "To the theoretical explanation of the universal response", Physica B 123 (1984) 739-745.

[23] R. R. Nigmatullin, "The realization of the generalized transfer equation in a medium with fractal geometry", Phys. Stat. Sol. B 133 (1986) 425-430. 
[24] I. Podlubny, Fractional differential equations (Academic Press, San Diego, CA, 1999).

[25] M. Raberto, E. Scalas and F. Mainardi, "Waiting-time and returns in high-frequency financial data: an empirical study", Physica A 34 (2002) 749-755.

[26] H. E. Roman and P. A. Alemany, "Continuous-time random walks and the fractional diffusion equation", J. Phys. A 27 (1994) 3407-3410.

[27] L. Sabatelli, S. Keating, J. Dudley and P. Richmond, "Waiting time distributions in financial markets", Eur. Phys. J. B 27 (2002) 273-275.

[28] A. I. Saichev and G. M. Zaslavsky, "Fractional kinetic equations: Solutions and applications", Chaos 7 (1997) 753-764.

[29] W. R. Schneider and W. Wyss, "Fractional diffusion and wave equations", J. Math. Phys. 30 (1989) 134-144.

[30] R. Schumer, D. A. Benson, M. M. Meerschaert and B. Baeumer, "Multiscaling fractional advectiondispersion equations and their solutions", Water Resources Res. 39 (2003) 1022-1032.

[3I] H. M. Srivastava, K. C. Gupta and S. P. Goyal, The H-functions of one and two variables with applications (South Asian, New Delhi, 1982).

[32] W. Wyss, "The fractional diffusion equation", J. Math. Phys. 27 (1986) 2782-2785.

[33] G. Zaslavsky, "Fractional kinetic equation for Hamiltonian chaos, chaotic advection, tracer dynamics and turbulent dispersion", Phys. D 76 (1994) 110-122. 